\title{
Exploiting the Electrical Properties of thin Films of Semiconducting Polymers
}

\author{
Roberto M. Faria and O.N. Oliveira Jr. \\ Instituto de Física de São Carlos, USP \\ C.P. 369, 13560-970 São Carlos, SP, Brazil
}

Received 22 May, 1998

\begin{abstract}
A brief review is provided of the ways electrical properties of semiconducting polymers can be exploited. Emphasis is given to the analysis of electrical conductivity data, in particular to the importance of interchain processes that must be considered along with intrachain processes for bulk conductivity. Among the large variety of possible applications of thin films of semiconducting polymers, the focus is placed on recent breakthroughs in producing diodes, field effect transistors and electroluminescent devices out of these materials. The fabrication of ultrathin films from semiconducting polymers, using the Langmuir-Blodgett and the self-assembly techniques, is discussed to illustrate the importance of processibility issues for any real application including these polymers.
\end{abstract}

\section{Introduction}

Due to their remarkable intrinsic electrical insulating characteristics, organic polymeric materials have been for a long time the subject of extensive dielectric and charge storage studies [1-3]. Electrical breakdown, charging-discharging mechanisms, thermoelectric and radiation effects, and dielectric relaxation processes have been investigated from both experimental and theoretical points of view. Insulating polymers are now widely used as insulation in power cables in energy distribution networks, and in different types of high accuracy equipment. The contribution of Prof. B. Gross in this area started at the early 70 s, when he introduced fluorinated polymers as extremely good electrets owing to their large charge storage capacity and remarkable mechanical properties [4]. The combination of such properties allowed these polymers to be applied as active membranes in electroacoustic devices [5]. Later on the family of polyvinylidene fluoride (PVDF) found several applications as piezoelectric and pyroelectric devices, deriving from their ferroelectric activity [6].

Until the early 1980s, most studies and applications using electrical properties of polymeric materials only exploited their insulating and charge storage capabilities. A new turn, both in science and technology, took place in the 1980s with the advent of the conducting and semiconducting polymers [7], with polyacetylene being their foremost representative in the initial studies [8]. Several possible applications have since been suggested and actually demonstrated in which the polymer is employed not only as a passive, insulating element, but also with an active role, as in microelectronic device components [9].

One major difficulty, which is even now a stumbling block for the widespread use of conducting polymers, is fabrication in the required form and shape. The first conducting polymers produced were almost invariably insoluble and infusible. Over the years, new approaches have been implemented by various research groups in order to overcome the fabrication difficulty. Such approaches stem from synthetic chemistry methods [10] with incorporation of side groups that make the polymer soluble, new techniques for growing films electrochemically [11] and the use of functionalized acids to improve solubility in standard organic solvents [12]. These major efforts have now paid off, which is demonstrated by the large number of conducting polymers produced worldwide, some of them commercially available [13].

It is not our intention to provide here a full review of the electrical properties and applications of semiconducting polymers. A vast literature on the subject already exists [14]. We shall restrict ourselves to the properties influencing processibility of conducting polymers for the fabrication of ultrathin films, such as the Langmuir-Blodgett (LB) [15-18] and self-assembled (SA) films [18-21], and to the analysis of electrical conductivity data. As for the possible applications of conducting polymers, we shall discuss the recent breakthroughs in producing diodes, FETs and electroluminescent devices. 


\section{Electrical conductivity of semiconducting polymers}

The singular - and most important - property of conjugated polymers is their electronic conduction along a single chain. Under doping, the conductivity of a polymeric film may increase by up to fifteen orders of magnitude, and the basic phenomenon causing this spectacular variation is the appearance of extended states in a chain. This starts with the generation of localized states in the forbidden gap of the one-dimensional electronic structure, which are associated with induced conformational defects combined with solitons, polarons and/or bipolarons. The electronic properties of these polymeric chains have opened up a new branch of investigation in condensed matter, as well as a new class of materials - the so-called electronic plastics.

Since the bulk structure of organic polymeric films is surely among the most complicated in condensed matter, obviously the electronic conduction mechanisms involved in the charge transport of conducting and semiconducting polymers are not as simple as described by the band model. In a simple picture one may split the electronic conduction in these materials into two contributions: i) along a single molecule and ii) interchain processes. This has been the subject of numerous investigations since the late $1970 \mathrm{~s}$. In the following, we present a brief, generalized description of the two processes.
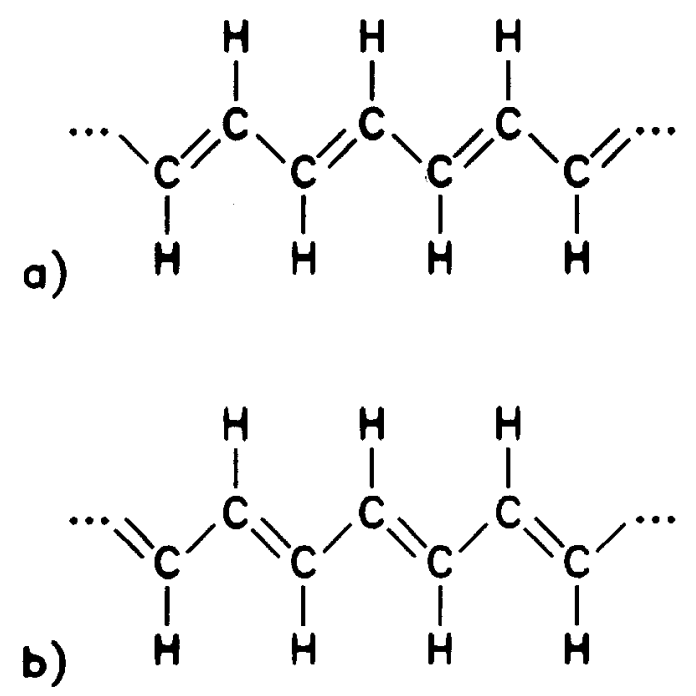

Figure 1. Two equivalent conformations for transpolyacetylene.

Theoreticians have been involved in the challenging task of establishing a framework for the single chain conductivity. The first widely successful model was the SSH model, developed by Su, Schrieffer and Heeger
$[22,23]$, in which the electrical conductivity is generated by solitonic defects in polyacetylene chains under doping. Because of its simple chemical structure, the transpolyacetylene molecule (infinite chain) was considered as the prototype of a conjugated molecule for molecular electronic conduction. Since trans-polyacetylene has two equivalent conformations (Fig. 1), the SSH model assumes a topological degenerate ground state $[22,23]$.

The SSH approach involves a tight-binding calculation for a single chain with cyclic boundary conditions, and neglects electron-electron interactions, with the chain being a sequence of carbon atoms in which $\sigma$-bonds are represented by a spring constant $K$. The Hamiltonian in the SSH model is then expressed by:

$$
\begin{gathered}
H=-\sum_{n}\left[t_{0}+\alpha\left(u_{n}-u_{n+1}\right)\right]\left(c_{n}^{+} c_{n+1}+c_{n+1}^{+} c_{n}\right)+ \\
\frac{1}{2 M} \sum_{n} p_{n}^{2}+\frac{K}{2} \sum_{n}\left(u_{n}-u_{n+1}\right)^{2} .
\end{gathered}
$$

where $c^{n+}$ and $c^{n}$ are the creation and annihilation operators, respectively. The first sum in the expression is the kinetic energy of $\pi$-electrons, where $t_{0}+\alpha\left(u_{n}-\right.$ $\left.u_{n+1}\right)$ is the linearized bond length dependence of the transfer integral $t_{n, n+1}, u_{n}$ is the displacement of the $n$-carbon atom, $t_{0}$ is the transfer integral of adjacent atoms when $u_{n}=0$, and $\alpha$ is the electron-phonon coupling constant. The second sum gives the kinetic energy of the lattice, where $p_{n}$ is the momentum of the atom at site $n$, and the third sum represents the $\sigma$ strain energy. A non-dimerized trans-polyacetylene chain may be considered as a one- dimensional $\pi$-electron system, with a half-filled electron band. It is therefore a onedimensional metallic system, which violates the Peierls instability [24]. The band structure for a non-dimerized system is represented by the dispersion relation $E(k)$ shown in Fig 2, while Fig. 3 presents a more realistic band structure for trans-polyacetylene incorporating bond dimerization. Dimerization generates an energy gap $2 \Delta$ in the dispersion relation at $k=\pi / 2 a$, where $a$ is the lattice parameter of a one-dimensional unit cell. 

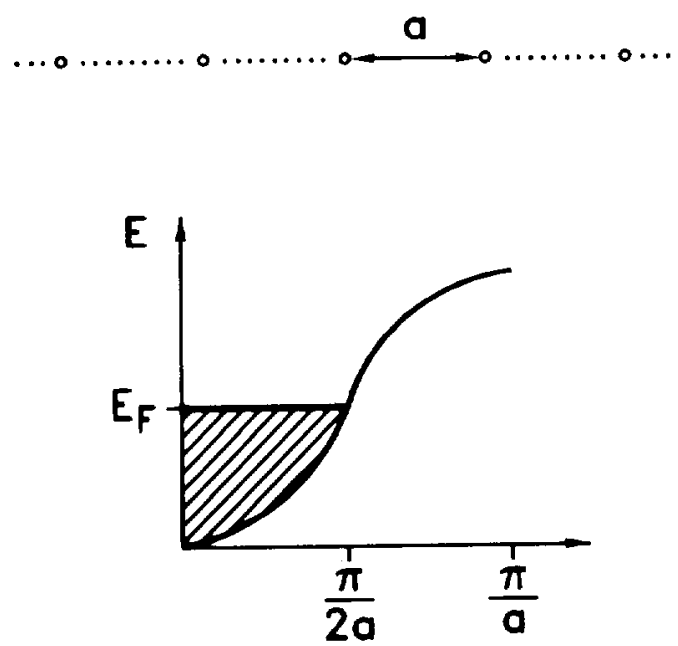

Figure 2. The dispersion relation $E(k)$ versus $k$ for a nondimerized system leads to a half- filled band. EF is the Fermi energy.
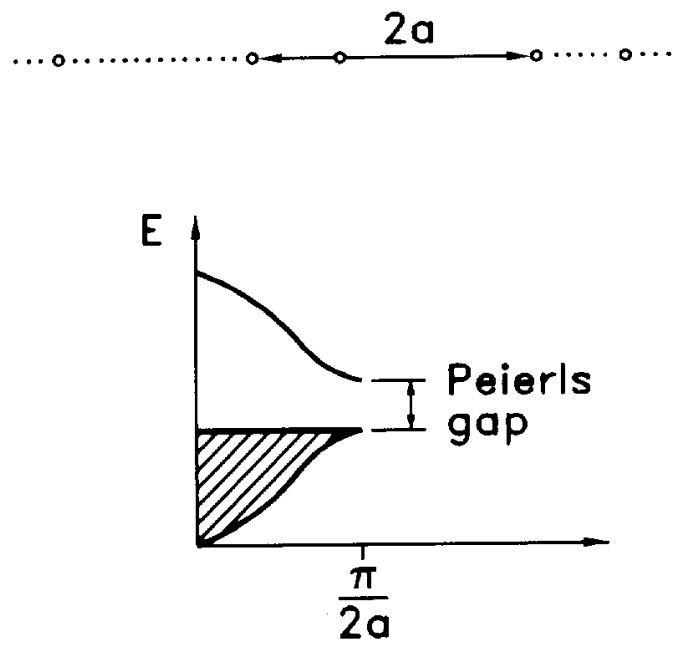

Figure 3. For a dimerized system, a gap appears in the $E(k)$ versus $k$ plot at the Fermi level, the so-called Peierls gap.

The bond alternation phenomenon (single-double to double-single sequences) in a trans-polyacetylene chain generates an electronic energy level in the mid-gap (see Fig. 4). This energy level, called a soliton, is spread over approximately 14 carbon sites and is of $p_{z}$-orbital nonbonding character. Under strong chemical doping, the number of such states increases significantly, generating an extended state configuration and consequently increasing the chain conductivity. In any other conjugated polymer each bond alternation generates a double level in the gap as shown in Fig 5.

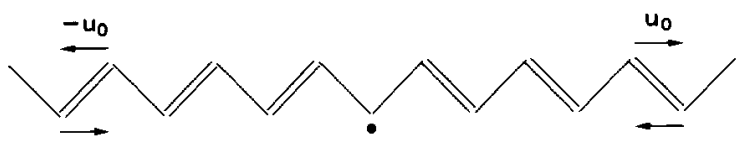

BC WIIIIIID

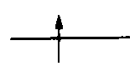

BV

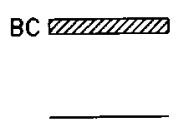

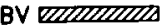

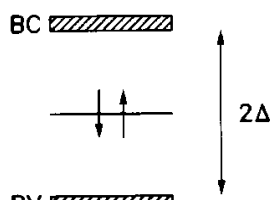

BV שIIIIIII
Figure 4. A defect, represented by a change in the alternation of the conjugated chain shown in the upper part of the figure, generates an electronic level within the gap. This electronic level is called a soliton. The gap energy is $2 \Delta$, corresponding to the $\pi-\pi^{*}$ gap. $\mathrm{BV}$ and $\mathrm{BC}$ are the valence and conduction bands, respectively. The figure on the left represents a neutral molecule with spin, the central figure corresponds to a spinless positively charged molecule, and the figure on the right a spinless negatively charged molecule.

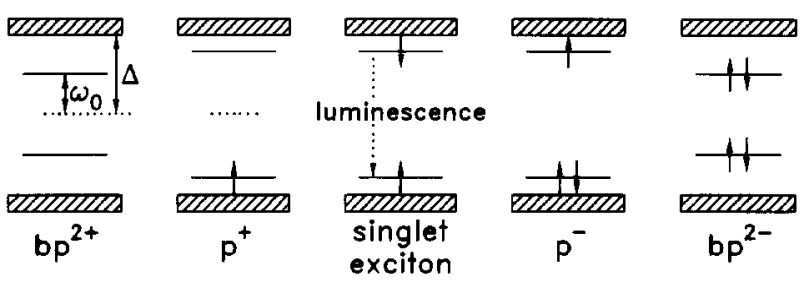

Figure 5. For conducting polymers other than transpolyacetylene, a defect in the bond alternation generates a double level in the $2 \Delta \mathrm{gap}$. In the figure, $b p^{2+}$ and $b p^{2-}$ stand for the bipolaronic levels while $p^{+}$and $p^{-}$stand for the polaronic levels. The figure in the middle represents the unstable exciton.

The levels in the forbidden band enhance the bulk conductivity of these highly disordered polymeric materials. The bulk conductivity also depends strongly on interchain processes, as illustrated in a study of ac conductivity of protonated polyaniline [25]. In the PANi structure, the dopant molecules are not uniformly distributed, but agglomerate forming dispersed conductive islands [26]. Nevertheless, it is reasonable to expect that traces of dopant molecules exist in the entire structure due to the diffusion process. The original (undoped) matrix remains less conductive, with carrier transport occurring via a hopping mechanism which seems to obey the random-free barrier model [27], and is therefore temperature dependent.

Within this model, the effective complex conductivity, $\tilde{\tilde{\sigma}}$ of a doped sample is expressed as [28] 


$$
\tilde{\sigma}(\omega)=i \omega\left[\epsilon_{2}^{\prime}-\frac{i \sigma_{2}^{*}(\omega)}{\omega}\right] \frac{2(1-\eta)\left[\epsilon_{2}^{\prime}-\frac{i \sigma_{2}^{*}(\omega)}{\omega}\right]-(1+2 \eta) \frac{i \sigma_{1}(\omega)}{\omega}}{(2+\eta)\left[\epsilon_{2}^{\prime}-\frac{i \sigma_{2}^{*}(\omega)}{\omega}\right]-(1-\eta) \frac{i \sigma_{1}(\omega)}{\omega}}
$$

where $f=\omega / 2 \pi$, and the indices 1 and 2 refer respectively to medium 1 , comprising conductive islands with a real conductivity $\sigma_{1}$, and medium 2 comprising the matrix with real conductivity $\sigma_{2}$. The dielectric constants of medium 1 and 2 are $\epsilon_{1}$ and $\epsilon_{2}$, respectively. The conductive islands are assumed spherical, with $\eta$ being their relative concentration in terms of volume fraction. Since not all the molecules in the conductive islands are strongly doped, $\eta$ is necessarily higher than the doping degree. The conductivity of the matrix, $\sigma_{2}(\omega)$, is assumed to occur by hopping in a disordered medium, governed by random free-energy barriers between the residence sites, and so obeying Dyre's equation [27].

$$
\tilde{\sigma}(\omega)=\sigma(0)\left[\frac{i \omega / \gamma_{\min }}{\ln \left(1+i \omega / \gamma_{\min }\right)}\right]
$$

$\gamma_{\text {min }}$ is activated following an Arrhenius process $\gamma_{0} e^{-W_{\min } / k T}$, where $\gamma_{0}$ is a frequency factor and $W_{i}$ the minimum value of the free-energy barriers. $\tilde{\sigma}_{D}(\omega)$ is essentially constant at low frequencies, i.e for $\omega / \gamma_{\min }<<$ 1 , but rises at $\omega / \gamma_{\min } \approx 1$. The physical reason for this is that at low frequencies the carrier has enough time to find a difficult jump, requiring a high activation energy. For increasing frequencies, the motion becomes more localized in time and the carrier has increasingly lower probability of facing a difficult jump. Hence the activation energy of the process tends to decrease.

Figures 6 and 7 show both the real and the imaginary components, $\sigma^{\prime}(f)$ and $\sigma^{\prime \prime}(f)$, of a.c. conductivity measured in PAN films, with different doping degrees and at different temperatures, respectively. The continuous curves are the fittings obtained from the model described by the combination of Eqs. (2) and (3). It is important to remark that the Kramers-Kronig relations, in the frequency range employed here, do not fully determine $\sigma^{\prime \prime}(f)$ out of $\sigma^{\prime}(f)$ and vice-versa. Related problems, derived from the non-local character of the Kramers-Kronig relations, have already been discussed in the literature [29].

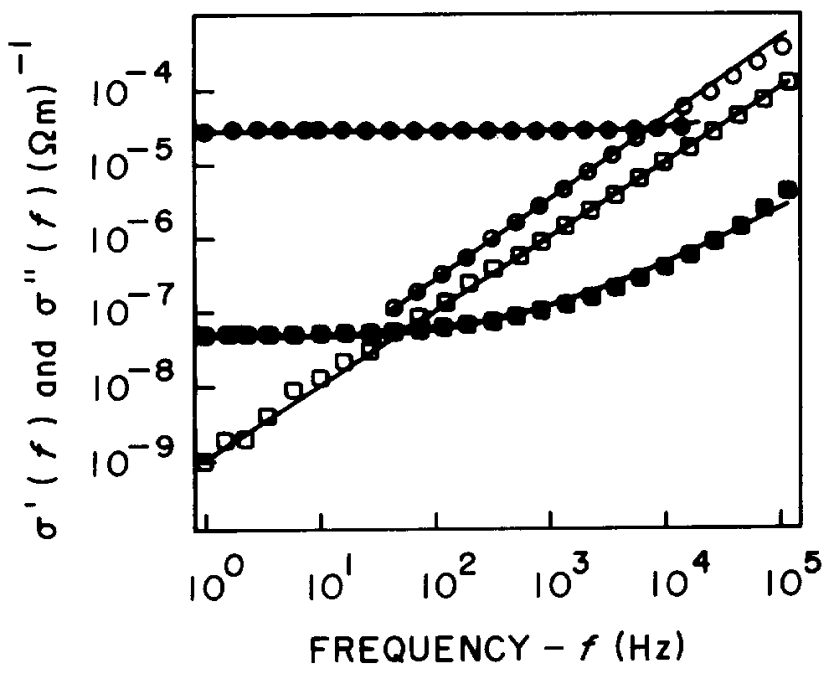

Figure 6 . The real and imaginary components, $\sigma^{\prime}$ and $\sigma^{\prime \prime}$, respectively, of the conductivity are plotted against the frequency, $f$, for two degrees of doping of a PAN film. The real component is represented by full circles (moderately doped) and full squares (weakly doped), while the imaginary component is represented by empty circles (moderately doped) and empty squares (weakly doped). The measurements were carried out at $300 \mathrm{~K}$.

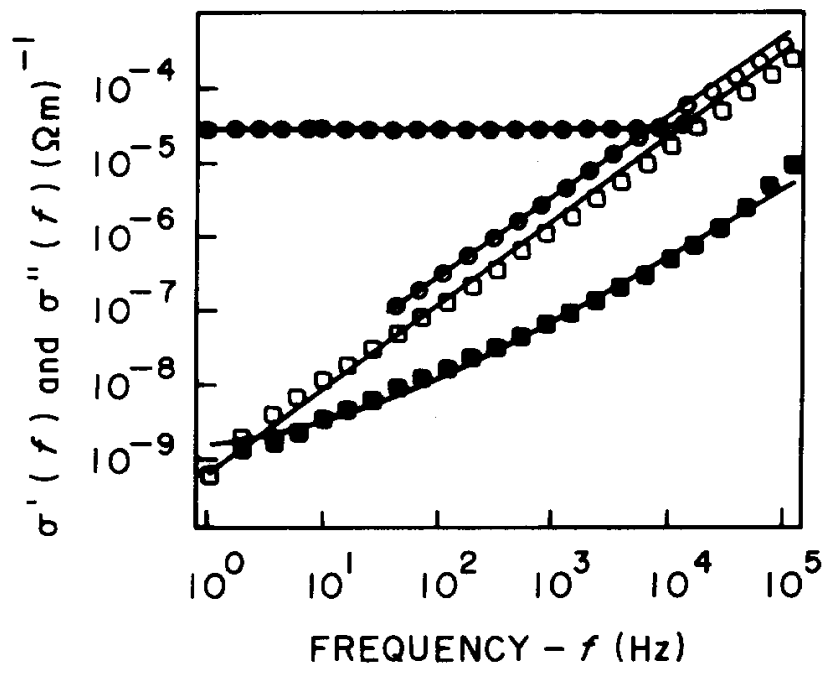

Figure 7 . The real and imaginary components, $\sigma^{\prime}$ and $\sigma^{\prime \prime}$, of the conductivity are plotted against the frequency, $f$, for two temperatures in a moderately doped PAN film. The real component is represented by full circles $(300 \mathrm{~K})$ and full squares (84), while the imaginary component is represented by empty circles $(300 \mathrm{~K})$ and empty squares (84K). 
The bulk conductivity is an important physical parameter not only because it monitors the doping efficiency of polymer molecules, which bear charged defects in the chains, but mainly because its value directly reflects three-dimensional charge delocalization phenomena. The complete conduction process involves the interchain diffusion rate, which depends on the interchain transfer integral $t_{\perp}$ and on the intrachain free time $\tau_{\|}$[30]. The transfer integral $t_{\perp}$ is related to the overlap of the electron wave function of two neighboring molecules and decays exponentially with increasing interchain distance. In summary, the successful application of the phenomenological model of a.c. conductivity is a clear indication that interchain processes must be taken into account when analyzing electrical properties of semiconducting polymers.

Further evidence of the importance of interchain (or interdomain) contribution to the measured electrical conductivity is provided by the analysis of experimental data from ultrathin polymer films. One should expect that Langmuir-Blodgett (LB) as well as self- assembling (SA) films made from conducting polymers should display higher conductivity owing to the organized nature of such films. However, measured d.c. conductivities for these ultrathin films are consistently lower than for their counterpart, spin-coated or dip- coated films [31]. The ultrathin films also contain islands, because of the tendency of conducting polymers to aggregate, and the aggregation in Langmuir monolayers can be probed at the air/water interface using Brewster angle microscopy [32]. The assumption of conducting islands was also employed to explain conductivity data on LB films of poly(3- hexylthiophene) [33]. The interdomain contribution may be the dominant one, because it is likely that any defect or pinholes, inevitable even in high quality, uniform LB films, may affect the connectivity between domains. This reduces the measured conductivity considerably, whereas for thicker films connectivity may not be a problem, in spite of the higher number of defects the films may possess.

\section{Examples of applications of semiconducting polymers}

Thin films of weakly and moderately doped conjugated polymers are suitable for some applications as active materials in electronic and photonic devices. Among the wide variety of possible applications that have been reported in the literature, we shall discuss diodes, electroluminescent devices and field-effect transistors (FETs).

\section{III.1 Diodes}

The physical principles as well as the processing requirements involved in electronic or photonic devices have as starting point the fabrication and characterization of a single diode. Two main rectifier systems have dominated microelectronics since early years: the p-n junction and Schottky diodes. When semiconducting polymers are employed as the active thin layers of new devices, interface phenomena play a major role, and therefore the study of such phenomena is the first, fundamental step in device research. Owing to its simplicity, a diode structure is adequate for a first study, and Schottky-barrier diodes are usually considered. The equation which governs the current across a Schottky interface is given by:

$$
J=J_{\text {sat }} \exp (e V / n k T)
$$

where $n$ is a factor taken as near unity for an ideal diode. However, in thin films tunneling injection through metal/semiconductor interfaces is often observed. At higher forward biases the current is limited by the bulk resistance, and in the case of thermionic emission the saturation current is $J_{\text {sat }}=A^{*} T^{2} \exp (e W / k T)$, where $A^{*}$ is the Richardson constant, equal to $120 \mathrm{~A} / \mathrm{K}^{2} \mathrm{~cm}^{2}$, and $W$ is the barrier height. This has been observed for diodes produced from poly(2-methoxy,5-(2'-ethyl-hexoxy)-1,4phenylene-vinylene), known simply as MEH-PPV [34]. In fact, several experiments were performed with a thin film diode of MEH-PPV, from which it could be unequivocally concluded that both hole and electron injection phenomena are dominated by tunneling at low biases, showing a Schottky barrier higher than $17 \mathrm{~V}$. Figure 8 shows that the $J-V$ characteristic curve of an ITO/MEH- PPV/Ca structure depends on the film thickness, but it is nearly thickness-independent for an $J-E$ ( $E$ is the electric field) characteristic curve. The device obeys a Fowler-Nordheim injection mechanism expressed by:

$$
J \propto E^{2} \exp (-K / E)
$$

where $K$ is a parameter that depends on the barrier shape, as shown in Fig. 8b. 

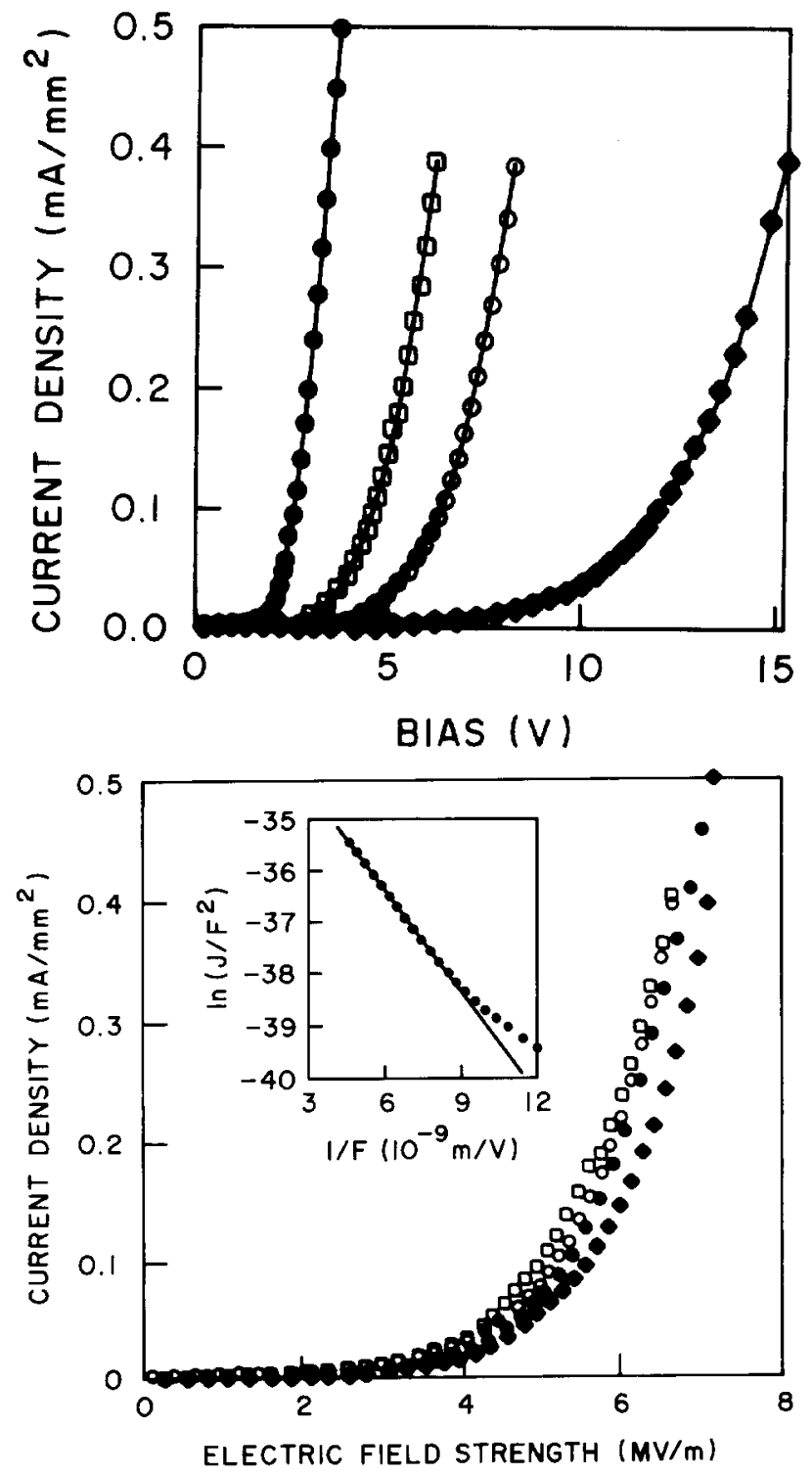

Figure 8. (a) Current density versus bias voltage characteristics for ITO/MEH-PPV/Ca structures of different thicknesses. Full circles - $47.5 \mathrm{~nm}$, Empty squares - $90 \mathrm{~nm}$, Empty circles $-120 \mathrm{~nm}$ and Full squares $-210 \mathrm{~nm}$. From ref. [34]. (b) Current density $(\mathrm{J})$ versus electric field (E) for the structures of (a). These curves are very nearly independent of thickness, as shown in the insert for one particular thickness. From ref. [34].

Another set of interesting experiments were carried out with poly(o- methoxyaniline) (POMA) samples doped with $\mathrm{HCl}$ on one surface [35]. Figure 9 shows a clear rectification effect, in which the protonated surface region, consisting mainly of the conducting emeraldine salt of poly(o-methoxyaniline) (POMA-ES), most probably acts as the contact injecting holes into the undoped bulk containing the emeraldine base poly(omethoxyaniline) (POMA-EB). This rectification phenomenon is also consistent with the hypothesis that holes are the mobile carriers in the POMA structure. Very similar effects were observed with other dopants
$\left(\mathrm{H}_{3} \mathrm{PO}_{4}\right.$ and toluene sulfonic acid, TSA $)$ and also with polyaniline (PAN) films [36]. The hole mobility in the POMA bulk was directly obtained from time-of-flight measurements, yielding $3 \times 10^{-4} \mathrm{~cm}^{2} / \mathrm{Vs}$, which is similar to that found in poly(p-phenylene vinylene) (PPV) [37]. Apparently, conjugated polymers possess similar carrier mobilities when interchain hopping processes prevail [38].

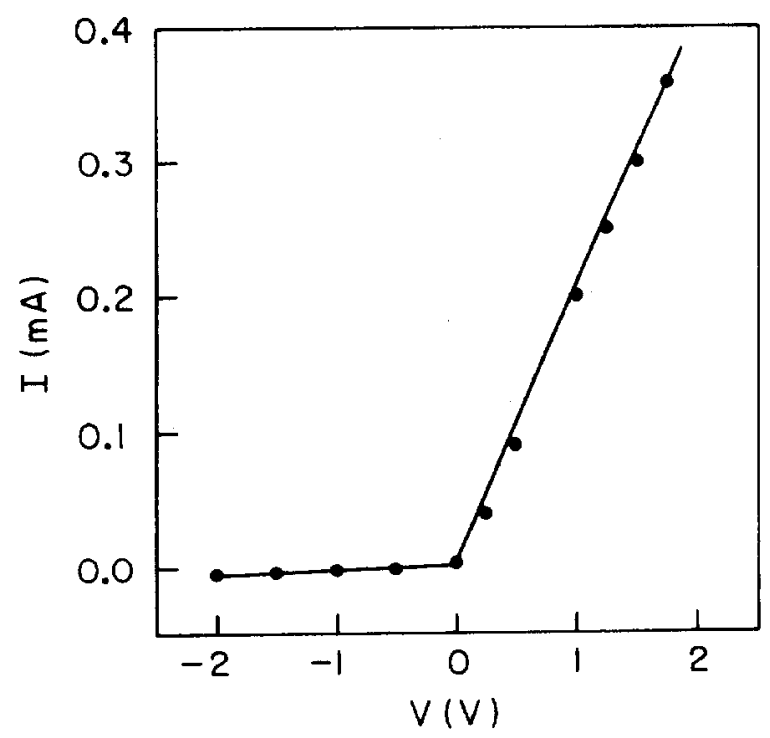

Figure 9. Current versus bias voltage characteristics for a poly(o-methoxyaniline) sample doped on one surface with HCl, showing rectification. From ref. [35].

\section{2 Electroluminescent devices}

A great deal of excitement has been generated in the recent past over the possibility of producing large area, flexible displays and even lasers employing electroluminescent polymers. The incredibly rich variety of materials that can be synthesized is a promising feature of these novel materials, of which the PPV family is perhaps the most important. By the same token, such a variety requires extensive efforts in research and development for the identification of: i) the most suitable material for a given application; ii) the most appropriate conditions under which this material is to be employed. The luminescence originates most probably from recombination involving the unstable excitonic level in the band gap (see Fig. 5). Polythiophenes, poly(paraphenylene), and poly(paraphenylene vinylidene) generate substantial luminescence, and diode structures have been produced using these materials. Fig. 10 shows photoluminescent and electroluminescent absorption spectra of PPV [9], while the $\mathrm{J}$ vs $\mathrm{V}$ and luminescence irradiation characteristics are presented in 
Fig. 11 for a luminescent diode ITO/MEH- PPV/Ca [34].

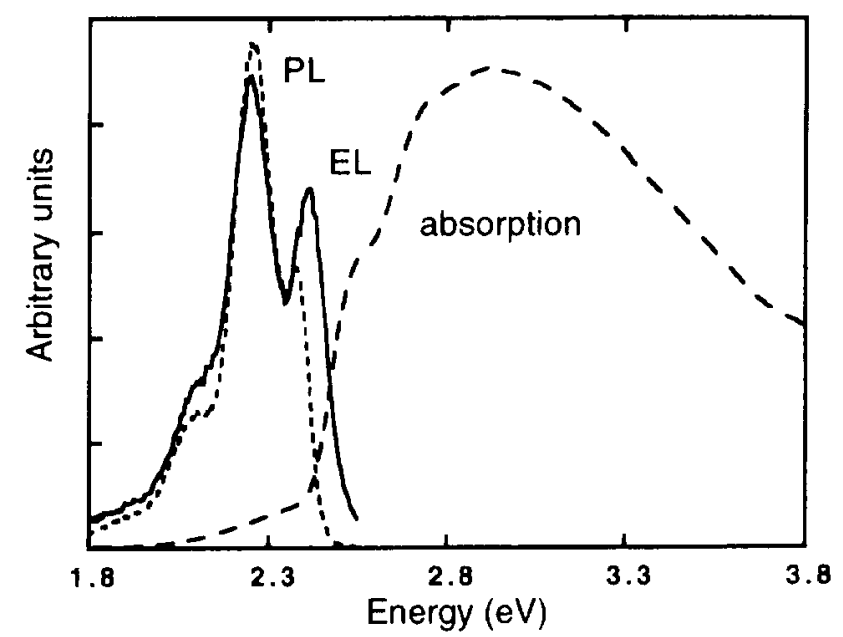

Figure 10. Absorption, photoluminescent emission and electroluminescent emission curves for PPV at $300 \mathrm{~K}$. From ref. $[9]$.

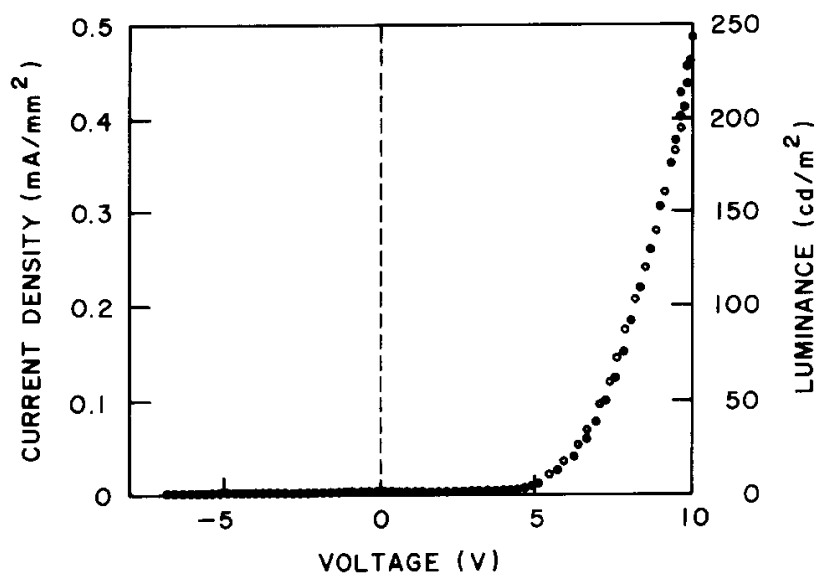

Figure 11. Rectification curve (full circles) of a diode of PPV and the corresponding luminescence curve (empty circles) as a function of the applied voltage. From ref. [34].

Since changes in the polymeric film, following modification of the polymer itself by chemical synthesis, or of the film architecture, may cause the luminescent properties to vary widely, the opportunity to tailor the materials for specific applications exists. Thus, for the same polymer family (e.g. PPV and their derivatives) the color of emission may be changed from yellow-greenish to orange [39] to blue [40]. Furthermore, combination of different polymers may also vary the emitted color [41]. Many researchers have sought an enhancement in performance by employing the polymeric materials in the form of ultrathin films. A certain degree of molecular control has already been demonstrated in the changes of fluorescence properties of SA films made from PPV [39]. In the latter work, the fluorescence emission from PPV was quenched by building adjacent layers of PPV/C60 (sulfonated fullerene), owing to the non-radiative electron transfer from PPV to C60 [39]. This quenching behavior was observed earlier [42] in cast films of PPV/C60. The novelty [39] was that the fluorescence could be recovered if inert spacer layers (say of polyallylamine and sulfonated polystyrene (PAH/SPS)) were interposed between PPV and C60 layers. This option is not available in the spin-coated films.

Using SA films, it has been possible to build devices whose emission wavelengths can be altered and whose efficiencies can be enhanced by altering the film architecture. This was well illustrated by Rubner and collaborators $[43,44]$, which showed that the light output of PPV SA films could be increased by identifying optimized supramolecular architectures that contained PPV/SPS (polystyrene sulfonic acid) and PPV/PMA (polymethacrylic acid) layers. The output could be further enhanced if passive layers of SPS/PAH (polyallylamine) were deposited adjacent to the aluminum electrode. This example again points to the advantage of employing materials whose properties can be tailored at the molecular level.

\section{III.3 FETs}

Here we provide a brief description of the fabrication process and characterization of FETs produced from poly(o-methoxyaniline), POMA. We believe that the results and their analysis illustrate the potential of conducting polymers and also the problems to be overcome for the successful application of these materials in devices. Fig 12 shows a field effect transistor of poly(o-methoxyaniline) (POMA-FET) where a highly doped p-silicon substrate, used as gate electrode, was covered with a thin, thermally grown $\mathrm{SiO}_{2}$ film. Both source and drain electrodes were made from evaporated gold with a finger shape, with length $\mathrm{L}$ and width $W$. Drain current-voltage characteristic curves ( $I_{D}$ vs $\left.V_{D}\right)$, for different gate voltages, $V_{G}$, are shown in Fig. 13 . The drain-source conductivity increases with negative $V_{G}$ values, indicating a p-type semiconductor as already demonstrated for a POMA film.

The characteristics of a FET device are usually represented by a plot of the drain current $I_{D}$ as a function of the voltage $V_{D}$, with the gate voltage $V_{G}$ as a parameter [45],

$$
I_{D}=C_{o x} \mu W / L\left[\left(V_{G}-V_{t h}\right) V_{D}-1 / 2 V_{D}^{2}\right]
$$

in which, for higher values of $V_{D}$, the saturation region is described by: 


$$
I_{D, s a t}=(W / 2 L) \mu C_{o x}\left(V_{G}-V_{t h}\right)^{2}
$$

$I_{D, s a t}$ is the drain current in the saturation regime, $V_{t h}$ is the threshold voltage, $V_{G}$ is the gate voltage, $\mu$ is the carrier mobility, and $C_{o x}$ is the capacitance per unit area of the oxide layer. The conductivity of the POMA film is given by $\sigma=n \mu e$, where $n$ is the carrier density and $e$ is the electronic charge. The results of Figure 13 may be fitted using $\mu=1.710^{-3} \mathrm{~cm}^{2} \mathrm{~V}^{-1} \mathrm{~s}^{-1}$, $V_{t h}=-7.5 \mathrm{~V}$ and $\sigma=310^{-8} \Omega^{-1} \mathrm{~cm}^{-1}$. In comparison with what is found in the literature, the mobility here is high for a conjugated polymer with low conductivity.

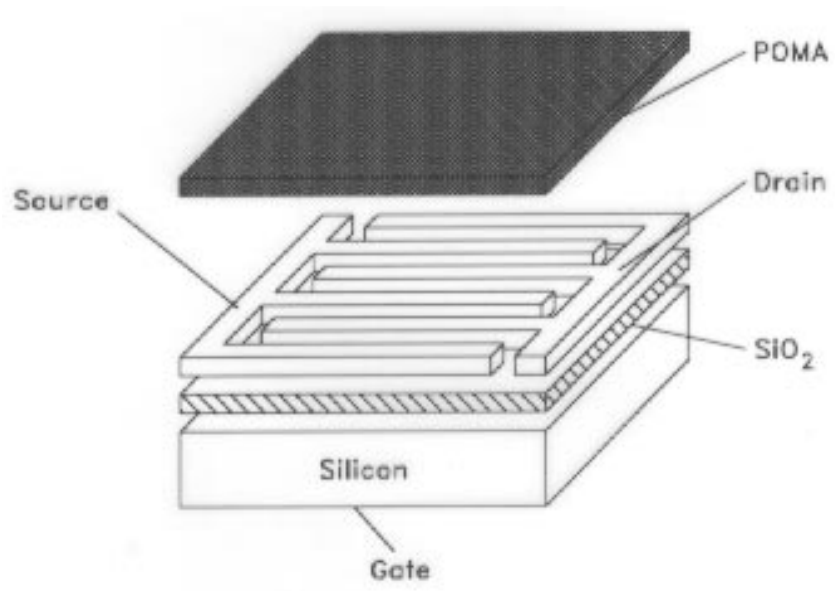

Figure 12. Schematic diagram of a field-effect transistor made from poly(o-methoxyaniline). A strongly p-doped silicon was used as gate, covered by a thin layer of $\mathrm{SiO}_{2}$. The drain and source electrodes were made from evaporated gold with a finger shape. A POMA layer is deposited through spin coating on top of this arrangement.

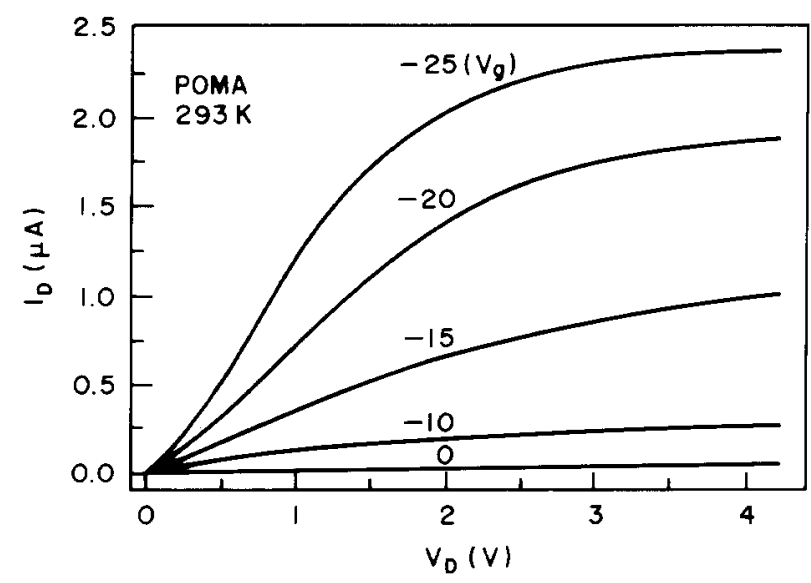

Figure 13. Drain current, $I_{D}$, versus drain voltage, $V_{D}$, for a POMA FET and different values of gate voltage, $V_{G}$.

\section{IV polymers}

In the last two sections, a number of results and examples were described in connection with thin films of conducting polymers, with no mention of the difficulties in producing such films. We now elaborate on production difficulties which have required intensive work not only in chemical synthesis but also in the engineering of conducting polymers. The focus will be on ultrathin films, namely Langmuir-Blodgett (LB) [15-18] and selfassembled (SA) films [18-21], since the requirements for producing these are usually the most stringent. In fact, the interest in these films appeared owing to the possibility of enhancing material properties by some degree of molecular control. This stems from the encouraging results obtained with conducting polymers processed in a number of different ways, based on a rich variety of materials and on the immense potential for applications.

In the Langmuir-Blodgett method, a very thin layer of the polymeric material, ideally a monolayer, is spread on an aqueous subphase which can then be transferred onto solid substrates in a layer-by-layer fashion. This method offers precise control over the film thickness, with ultrathin (nm) films being produced, and the possibility of tailoring the film properties by altering the film architecture. Changes in architecture can be performed at two levels, either by modifying the polymer backbone or attaching side groups to the chains, and/or in the fabrication of superstructures similar to the semiconductor heterostructures produced by molecular beam epitaxy, in which two or more materials are employed.

A large variety of conducting polymers have been used for LB film fabrication, as pointed out in a recent review of the subject [31]. The main classes of polymers include: polyaniline, polypyrroles, polythiophenes, poly(p-phenylene vinylene) (PPVs) and polyacetylenes. The first step in producing good quality LB films is to obtain and characterize the so-called Langmuir monolayer, and the initial difficulty is always associated with the solubility of the conducting polymers. Another characteristic feature of conducting polymers is that the monolayers spread from these materials are generally not true monolayers. It is likely that stable multilayer stacks are formed, and therefore, unlike the traditional amphiphilic compounds used in LB technology, the monolayer characteristics of the conducting 
polymers depend upon experimental conditions such as volume of solution spread, concentration of the spreading solution and even speed of barrier compression.

Such dependency arises in most cases from the tendency of conducting polymers such as polyaniline and its derivatives to form aggregates [46]. As a consequence, optimized experimental conditions for obtaining stable monolayers leading to high quality LB films must be determined. By way of illustration, we mention a systematic study [47] in which several mixtures of functionalized acids and organic solvents were tried to render the parent polyaniline soluble, which could then be used to form stable Langmuir monolayers. Stability and transferability were only achieved if the parent polyaniline was doped while on the aqueous subphase, requiring the use of low $\mathrm{pH}$ subphases [48]. Other approaches which have been employed for producing LB films from polyanilines involve attaching long alkyl chains [49] or alkoxy groups [50] to the polymer backbone. The resulting polyanilines are in some cases readily soluble in various solvents, thus allowing the formation of LB films. Yet another method uses mixed monolayers in which the polymer is co-spread on an aqueous subphase with a film-forming material such as the amphiphilic fatty acids [51]. These mixed monolayers are readily transferred, leading to excellent film uniformity [52].

Incorporation of long alkyl chains into polythiophene chains has also been extensively used for producing LB films [53]. In some cases, the mixed monolayer approach was also employed, the modified polythiophene being spread on an aqueous surface together with fatty acids [54]. Such films could be doped with $\mathrm{NOPF}_{6}$, and used as the semiconducting element in field efffect transistors (FETs) [33]. Polypyrroles are also extremely promising materials, because of their electrical properties. Producing LB films from polypyrrole required until very recently very ingenious engineering methods, e.g. LB films have been formed using in situ polymerization [55] or by employing alkyl pyrrole monomers [56]. An exciting new possibility has emerged recently with the advent of soluble polypyrrole [57]. This material is obtained by chemical synthesis employing functionalized acids, in a similar fashion to the solubility induced in polyanilines as described above. The fabrication of LB films from the parent polypyrrole and mixed films with fatty acids has been demonstrated [58]. The optical properties are very similar to those obtained with other polypyrrole films, but the conductivity is still relatively low, as has been the case for LB films from other conducting polymers. For the PPV family, processibility is also of extreme importance since PPVs are not soluble in organic solvents. Therefore ultrathin films must be formed from soluble PPV precursors which are then converted into PPV, usually by thermal treatment [59]. PPV films have been used in a variety of applications, such as in electroluminescent devices [60]. The advantages associated with the LB technology are also applicable to the SA films, for which molecular control can be achieved [61]. In the SA technique, alternating layers of cationic and anionic polymers are adsorbed on a solid substrate [19]. Furthermore, the SA technique has an important advantage in that it does not require sophisticated equipment. The LB and SA techniques may be considered as complementary.

Polymers varying from the widely studied polyanilines and other conducting polymers to various polyelectrolytes have been employed and a number of potential applications have been considered [61]. The most investigated and perhaps the most important one in the foreseeable future is in electroluminescent devices [43,44,62], as discussed in Section III. The actual development of any real world application is always preceded by systematic studies to establish property-structure relationships. For the SA films, in particular, there are a number of issues to be addressed, starting with the process of adsorption itself. Determining the ideal time period for adsorption of a layer of a given material, and how the adsorption rate varies under different experimental conditions are tedious but necessary tasks. Within this rationale, a systematic investigation was carried out on the kinetics of adsorption of a polyaniline derivative (POMA) onto glass substrates [63]. It was found that the adsorption occurs in two steps: a very fast, first order kinetics process with characteristic times of $5-10 \mathrm{~s}$, followed by a much slower process (characteristic time of $\sim 100 \mathrm{~s}$ ), indicative of a JohnsonMehl-Avrami-type adsorption. These processes correspond to nucleation of domains in the first stage, followed by domain growth in the second stage, as shown by atomic force microscopy [63].

The amount of material adsorbed as well as the kinetics of adsorption are also affected by other experimental conditions, such as the $\mathrm{pH}$ and polymer concentration $[64,65]$. For the POMA system, for instance, adsorption isotherms have shown that adsorption is mainly an entropy-controlled process, with the 
amount of material adsorbed varying according to a simple Langmuir isotherm. It is also worth stressing that even though the SA technique for conjugated polymers was initially entirely based on the electrostatic interaction, with adsorption of alternating polycationic and polyanionic layers, it is now possible to build high quality SA layers employing H-bond interactions [39].

\section{Concluding Remarks}

In this paper we have focused on two important aspects of the exploitation of semiconducting polymers in real world applications, namely the ability to build supramolecular structures from these materials, and the analysis of electrical properties of semiconducting polymer films. Recent breakthroughs in fabrication techniques have made it possible to produce ultrathin films of a variety of semiconducting polymers. However, owing to the rich variety of new materials that can be produced, novel developments in the fabrication of films are likely to be an important issue for some time to come. With regard to the electrical properties, we have shown that interchain processes must be taken into account when analyzing the bulk electrical conductivity of semiconducting polymer films. This was illustrated by the application of a phenomenological model that includes carrier transport via an interchain hopping mechanism. Polyanilines, in addition, were considered as comprising conducting islands dispersed in a less conductive, undoped matrix. Possible applications of the semiconducting polymers were also discussed, by taking specific examples in the fabrication of diodes, field-effect transistors and electroluminescent devices.

\section{Acknowledgements}

The authors are thankful to Dr. R.K. Onmori and C.F.O. Graeff for helpful discussions. They are also indebted to FAPESP and CNPq for financial support.

\section{References}

1. G.M. Sessler, Electrets, Topics in Applied Physics Series, Vol. 33 (Springer, Heidelberg, 1987).

2. J. Lewiner, D. Morisseau and C. Alquié (Eds), Proceedings 8th International Symposium on Electrets, IEEE Catalog. No. 94 CH3443-9, (IEEE, N. York, 1994).

3. R. Gerhard-Multhaupt and A.S. DeReggi (Eds), IEEE Transactions on Dielectrics and Electrical Insulation 3, 601 (1996).
4. B. Gross, G.M. Sessler and J.E. West, J. Appl. Phys. 47, 968 (1976).

5. B. Gross, in "Static Electrification", Institute of Physics, London, pp. 33-43 (1971).

6. T. Furukawa, Phase Transitions 18, 143 (1989).

7. J.L. Brédas and R. Silbey, Conjugated Polymers, (Kluwer Academic Publishers, Boston, 1991).

8. C.K. Chiang, J.C.R. Fincher, Y.W. Park, A.J. Heeger, H. Shirakawa, E..J. Louis, S.C. Gau, A.G. MacDiarmid, Phys. Rev. Lett. 39, 1098 (1977).

9. N.C. Greenham and R.H. Friend, in Solid State Physics Series, Vol. 49, ed. by H. Ehrenreich and F. Spaepen, (Academic Press, San Diego, 1995, pp 1-149).

10. L. Robitaile, J-Y. Bergeron, G. D’Aprano, M. Leclerc and C.L. Callender, Thin Solid Films 244, 728 (1994).

11. A.A. Athawale, S.F Patil and B. Deore, Polymer International 45, 195 (1998).

12. Y. Cao, P. Smith and A.J. Heeger, Synth, Met. 55-57, 3514 (1993).

13. Y. Cao, G.M. Treacy, P. Smith and A.J. Heeger, Synth. Met. 55-57, 3526 (1993).

14. H.S. Nalwa (Ed.), Handbook of Organic Conductive Molecules and Polymers, Vol. 4, Conductive Polymers: Transport, Photophysics and Applications (John Wiley, N. York, 1997).

15. G.L. Gaines Jr., Insoluble monolayers at LiquidGas Interface, (Interscience, New York, 1966).

16. G.G. Roberts, Langmuir-Blodgett Films, (Plenum Press, New York, 1990).

17. M.C. Petty, Langmuir-Blodgett Films - An Introduction, (Cambridge University Press, Cambridge, 1996).

18. A. Ulman, An Introduction to Ultrathin Organic Films - from Langmuir-Blodgett to Self-Assembly, (Academic Press, New York, 1991).

19. G. Decher, J.D. Hong and J. Schmitt, Thin Solid Films 210/211, 831 (1992).

20. Y. Lvov, G. Decher and H. Möhwald, Langmuir 9, 481 (1993).

21. M. Ferreira, J.H. Cheung and M.F. Rubner, Thin Solid Films 244, 806 (1994).

22. W.P. Su, J.R. Schrieffer and A.J. Heeger, Phys. Rev. Lett. 42, 1689 (1979).

23. W.P. Su, J.R. Schrieffer and A.J. Heeger, Phys. Rev. B 22, 2099 (1980).

24. R. Peierls, Quantum Theory of Solids, (Oxford University Press, Oxford, 1955).

25. J.M. Ginder, A.F. Richter, A.G. MacDiarmid and A.J. Epstein, Solid State Commun. 63, 97 (1987).

26. D. Jeon, J. Kim, M.C. Gallagher and R.F. Willis, Science 256, 1662 (1992).

27. J. C. Dyre, J. Appl Phys. 64, 2456 (1988).

28. C.M. Lepienski, R.M. Faria and G.F. Leal Ferreira, Appl. Phys. Lett. 70, 1906 (1997).

29. H. Silva and B. Gross, Phys. Rev. 60, 684 (1941). 
30. Z.H. Wang, H.S. Javadi, A. Ray, A.G. MacDiarmid and A.J. Epstein, Phys. Rev. B 42, 5411 (1990).

31. L.H.C. Mattoso, O.N. Oliveira Jr. and M.Ferreira, Conducting Polymers (for LangmuirBlodgett Film Fabrication), Polymeric Materials Encyclopedia, J.C. Salamone (ed.), Vol. 2C, (CRC Press, Boca Raton, FL, 1432 1996).

32. A. Riul Jr., H. Haas, A. Dhanabalan, M.A. Cotta and O.N. Oliveira Jr., 4 ' Congresso Brasileiro de Polímeros, Salvador, Bahia (Brazil) (ABPOL, São Carlos, 1997).

33. E. Punkka, M.F. Rubner, J.D. Hettinger, J.S. Brooks and S.T. Hannahs, Phys. Rev. B, 43, 9076 (1991).

34. I. D. Parker, J. Appl. Phys. 75, 1656 (1994).

35. S. Mergulhão, R.M. Faria, G.F. Leal Ferreira and J. Sworakowski, Chem. Phys. Lett. 269, 489 (1997).

36. R.M. Faria and S. Mergulhão, unpublished results

37. M. Takiguchi, D.H. Park, H. Ueno, K. Yoshino and R. Sugimoto, Synth. Met. 17, 657 (1987).

38. E.M. Conwell, H.Y. Choi and S. Jayadev, Synth. Met. 49-50, 359 (1992).

39. M. Ferreira, O. Onitsuka, W.B. Stockton and M.F. Rubner, ACS Symposium Series, 672, 437 (1997).

40. Y. Kim, S. Kwon, D. Yoo, M.F. Rubner and M.S. Wrighton, Chem. Mat. 9, 2699 (1997).

41. J.A. Osaheni and S.A. Jeneke, Macromolecules 27, 739 (1994).

42. N.S. Sariciftci, L. Smilowitz, A.H. Heeger and F. Wudl, Science 258, 1474 (1992)

43. O. Onitsuka, A.C. Fou, M. Ferreira, B.R. Hsieh and M.F. Rubner, J. Appl. Phys. 80, 4067 (1996).

44. A.C. Fou, O. Onitsuka, M. Ferreira, M.F. Rubner and B.R. Hsieh, J. Appl. Phys. 79, 7501 (1996).

45. S. M. Sze, Physics of Semiconductor Devices, (Wiley, N. York, 1981).

46. S. Paddeu, M. K. Ram and C. Nicolini, J. Phys. Chem. B.101, 4759 (1997).

47. A. Riul Jr., L.H.C. Mattoso, G.D. Telles, P.S.P. Herrmann, L.A. Colnago, N.A. Parizotto, V. Baranauskas, R.M. Faria and O.N. Oliveira Jr.,
Thin Solid Films 284-285, 177 (1996).

48. A. Riul Jr. L.H.C. Mattoso, S.V. Mello, G.D. Telles and O.N. Oliveira Jr., Synth. Met. 71, 2067 (1995).

49. M. Ando, Y. Watanabe, T. Iyoda, K. Honda and T. Shimidzu, Thin Solid Films 179, 225 (1989).

50. L.H.C. Mattoso, S.V. Mello, A. Riul Jr., O.N. Oliveira Jr. and R.M. Faria, Thin Solid Films 244, 714 (1994).

51. A. Dhanabalan, R.B. Dabke, S.N. Datta, N. Prasanth Kumar, S.S. Major, S.S. Talwar, A. Q. Contractor, Thin Solid Films 295, 255 (1997).

52. (a) A. Dhanabalan, A. Riul Jr., L.H.C. Mattoso and O.N. Oliveira Jr., Langmuir 13, 4882 (1997). (b) A. Dhanabalan, A. Riul Jr. and O.N. Oliveira Jr., Supramolecular Sci., 5, 75-81 (1998).

53. I. Watanabe, K. Hong and M.F. Rubner, Langmuir 6, 1164 (1990).

54. A. Pawlicka, R.M. Faria, M. Yonashiro, S.V. Canevarolo, Jr. and O.N. Oliveira, Jr., Thin Solid Films 244, 723 (1994).

55. T. Shimidzu, T. Iyoda, M. Ando, A. Ohtani, T. Kaneko and K. Honda, Thin Solid Films 160, 67 (1988).

56. K. Hong and M.F. Rubner, Thin Solid Films 179, 215 (1989).

57. J.Y. Lee, D.Y. Kim, C.Y. Kim, Synth. Met. 74, $103(1995)$.

58. A. Dhanabalan, S.V. Mello and O.N. Oliveira Jr., Macromolecules 31, 1827 (1998).

59. D.D.C. Bradley and R.H. Friend, J. Phys. C: Condens. Matt. 1, 3671 (1989).

60. Y.Q. Liu, Q.L. Li, Y. Xu, X.Z. Jiang and D.B. Zhu, Synth. Met. 85, 1279 (1997).

61. G. Decher, Science 277, 1232 (1997).

62. R. Osterbacka, A..J. Pal, K.M. Kallman and H. Stubb, J. Appl. Phys. 83, 1748 (1998).

63. M. Raposo, R.S. Pontes, L.H.C. Mattoso, Macromolecules 30, 6095 (1997).

64. L.H.C. Mattoso, L.G. Patterno, S.P. Campana and O.N. Oliveira Jr., Synth. Met. 84, 123 (1997).

65. M. Raposo, L.H.C. Mattoso and O.N. Oliveira Jr., Thin Solid Films, 327-329, 739 (1998). 